\title{
Science on television: how? Like that!
}

\section{P. Maeseele and L. Desmet}

\begin{abstract}
This study explores the presence of science programs on the Flemish public broadcaster between 1997 and 2002 in terms of length, science domains, target groups, production mode, and type of broadcast. Our data show that for nearly all variables 2000 can be marked as a year in which the downward spiral for science on television was reversed. These results serve as a case study to discuss the influence of public policy and other possible motives for changes in science programming, as to gain a clearer insight into the factors that influence whether and how science programs are broadcast on television. Three factors were found to be crucial in this respect: 1) public service philosophy, 2) a strong governmental science policy providing structural government support, and 3) the reflection of a social discourse that articulates a need for more hard sciences.
\end{abstract}

\section{Introduction}

Research concerning science on television has greatly multiplied in recent years. ${ }^{1,2,3,4,5,6,7,8,9}$ However, there has been relatively little to no analysis of the factors that influence whether and how science programs are broadcast on television. This study explores the presence of science programs on Flemish television (the Dutch-speaking northern region of Belgium) between 1997 and 2002 in terms of length, science domains, target groups, production mode, and type of broadcast. Science programs on commercial television networks VMMA (Vlaamse Media Maatschappij) and SBS Belgium (Scandinavian Broadcasting System), however, appear to be absent. A spokesperson for the largest commercial broadcaster provides the following explanation:

\begin{abstract}
A commercial channel is tied to the choice of its viewers. Television is too expensive unless it attracts a sufficient number of viewers. For that reason, television is not fit for complicated scientific theories without direct social or human impact. ${ }^{10}$
\end{abstract}

Because science programs are absent on the commercial networks, our data collection is limited to the public broadcaster VRT (Flemish Radio and Television Network). These results serve as a case study to discuss the influence of public policy and other possible motives for changes in science programming. The aim of this paper is to gain a clearer insight into the factors that influence whether and how science programs are broadcast on television. Our data show that for nearly all variables 2000 can be marked as a year in which a decadelong downward spiral for science on television was reversed. Three factors were found to be crucial in this respect. First, a traditional emphasis of public service philosophy on education clearly distinguishes public broadcasters from other television networks. Second, a strong governmental science policy providing structural government support ensures the survival of, and may help to find a creative impetus for, science on television. And finally, the reversal in 2000 reflects a social discourse that increasingly articulates a need for more hard sciences.

\section{Method}

Since the commercial stations fail to broadcast science programs, this study only includes VRT data. The data on the programs' length and nature were put at our disposal by the VRT's research group. All of the public broadcasting company's programs are systematically analyzed according to a number of dimensions by means of the ESCORT (2.4) codes of the EBU (European Broadcasting Union). These classify all broadcasts according to several dimensions (content, intention, format, etc.). Biltereyst ${ }^{1}$ has emphasized that is very important to make a distinction between the content and the intention of a program. Science programs are often categorized as having an educational intention while science television could just as well serve an informative or entertaining intention. Therefore, it is vital to use the content dimension: "Science television then refers to 
programs that could have informative, educational or other (often mixed) intentions but from a content point of view are based on scientific facts, knowledge and developments". The content dimension allows distinguishing between nine categories when it comes to a scientific content: five categories for hard sciences and three for human sciences, which are described as those disciplines which primarily have a cultural character. Concerning the latter we find the categories language, literature, history, and a general category "other/mixed". For the hard sciences, we find the categories physical sciences (astronomy, chemistry, electricity, mechanics, physics, etc.), natural sciences (biology, botany, geology, zoology, etc.), applied sciences (dealing with material phenomena or industrial processes), medical sciences (medical and health subjects), and lastly, also a category "other/mixed", for those programs which were hard to classify. For this study, only the programs which are classified as one of these nine categories were taken into account. This effectively means that only science programs are included, i.e. programs whose content is entirely devoted to one of the nine categories, either in the format of documentary series, magazines, animated drama, etc., but not news bulletins or talk shows which happen to include a scientific item or scientific debate. A flagship science program for the public broadcaster has been How? Like that! (Hoe?Zo!), which was broadcast for five seasons on Sunday evenings on the first channel, which is a prime time television slot. This was an enormously successful science show, which immediately reached a market share of $46 \%$, and in which two famous Flemings needed to answer scientific questions in the presence of a panel of scientists. Although being devoted to an "other/mixed" hard science content, it was classified not as having an educational intention, but a general enrichment intention, as well as having a magazine format (for the subclass non-fiction in the format dimension). A second flagship has been the documentary series OverLeven ("On Life") which was broadcast on the second channel on prime time Sunday evenings, and which, amongst others, received the 2005 Descartes Prize for Science Communication from the EU commissioner for Science and Research for being an innovative science TV series which follows the work and personalities of scientists that solve key scientific enigmas. The program on average attracted 120.000 viewers (on a population of approximately six million). OverLeven has (also) been classified as having a general enrichment intention, a documentary format, but, dependent on the topic, as having either an applied, natural, physical, medical or "other/mixed" hard science content, or a historical content.

This study starts off in 1997, when a completely new legal framework for the public broadcasting company was set up. ${ }^{11}$ The BRTN (Belgian Radio and Television - Dutch-language broadcasts) was renamed to VRT, which from this moment on (and by means of a management contract) would be directed like a 'normal business company' in terms of 'industry efficiency'. At the same time both the structure and the operation of the broadcasting network was thoroughly changed by the channel profiling: TV1 (the broadening, popularizing channel that in 2005 was renamed to 'Eén'), Canvas (until autumn 1997 called TV2, the deepening, investigative channel for viewers looking for added value or sports) and Ketnet (for the youngest). In this study we will quantitatively analyze the first six years. These findings should allow us to identify the consequences of any new production strategies. It needs to be remarked that our data collection ends with 2002 as the research project in which these were collected ended shortly after.

With regard to other recent studies we are limited to Willems and Hanssen ${ }^{8,9}$ and Biltereyst. ${ }^{1}$ Yet, based on these studies it is perfectly possible to highlight a number of expectations. Willems and Hanssen ${ }^{8,9}$ found for 1991 that the second channel (at that time named BRT2) showed substantially more science than the main channel (BRT1), with a particular focus on natural sciences. Biltereyst ${ }^{1}$ has put this in a historical perspective; he proved that starting in 1989 (after the commercial channels started to broadcast in Flanders) science on the Flemish public broadcasting network ended up in a downward spiral.

\section{Results}

\section{Volume of science programs on VRT}

Table 1 lists the science volume on the VRT, grouped according to channel. From 1997 through 1999 the renewed mission and re-profiling caused both an absolute and relative decrease of the total share of science programs, with in 1999 a minimum number of 208.54 broadcast hours (which represents $2.97 \%$ of the overall broadcast time), a reduction of about one third. An opposite movement starts in the year 2000 where an uninterrupted increase of the broadcast time and share in the entire programming can be noticed. The absolute figures show that in 2001 the total science volume of 1997 is exceeded. But in relative figures we find that the total number of broadcast hours of the public station increases 
comparatively more than the number for science programs. The science share in the total broadcast time amounts to $4.33 \%$ in 2002 , which is still less than the $5.23 \%$ share in 1997 . This last conclusion dims a possible feeling of euphoria related to the absolute increase in the number of science broadcast hours.

\begin{tabular}{|ccclcc|}
\hline & TV1(h) & TV2/Canvas/Ketnet(h) & VRT(h) & VRT (\%) & Total broadcast time (h) \\
$\mathbf{1 9 9 7}$ & 117.29 & 185.65 & 302.94 & 5.23 & 5797 \\
$\mathbf{1 9 9 8}$ & 86 & 148.86 & 234.86 & 3.51 & 6685 \\
$\mathbf{1 9 9 9}$ & 32.87 & 175.67 & 208.54 & 2.97 & 7026 \\
$\mathbf{2 0 0 0}$ & 53.86 & 187.43 & 241.29 & 3.38 & 7147 \\
$\mathbf{2 0 0 1}$ & 103.31 & 232.07 & 335.38 & 4.44 & 7549 \\
$\mathbf{2 0 0 2}$ & 109.88 & 247.67 & 357.55 & 4.33 & 8251 \\
\hline
\end{tabular}

Table 1. Length and share of science programs on vrt, 1997-2002 (in hours and percentages).

The same tendencies can be seen when the data are grouped according to channel, except that we find TV2/Canvas/Ketnet's share growing already in 1999 (see table 1). This fact indicates a policy of an imposed distinction between the two channels, which also influences the science programming (see tables 2 and 3). The calculation of the number of science productions simply confirms the aforementioned trend. The number of programs on TV1 decreases from 210 in 1997 to 67 in 1999 and increases again in 2000 (108 productions). Concerning tv2/Canvas/Ketnet we find a decrease from 468 in 1997 to 226 in 1998, but in 1999 we find a significant increase to 442 programs. In 2002 there is a remarkable relapse concerning the number of broadcasts although the number of minutes keeps going up. From this we conclude that the average length of the programs on tv2/Canvas/Ketnet has increased in 2002.

\begin{tabular}{|c|c|c|c|c|c|c|}
\hline Hard Sciences & 1997 & 1998 & 1999 & 2000 & 2001 & 2002 \\
\hline Applied & $47.80(1)$ & & & & & \\
\hline Natural & 3890.58(89) & $2317.76(67)$ & $786.58(34)$ & $2937.46(102)$ & $6048.95(163)$ & $5846.46(154)$ \\
\hline Physical & $49.85(1)$ & & & & & \\
\hline Medical & $1038.88(57)$ & $62.05(2)$ & $30.83(1)$ & & & \\
\hline Other/mixed & $1596.80(54)$ & $2080.31(74)$ & $509.45(20)$ & & & $360.35(10)$ \\
\hline Total & $6623.91(202)$ & $4460.12(143)$ & $1326.86(55)$ & 2937.46(102) & $6048.95(163)$ & $6206.81(164)$ \\
\hline \multicolumn{7}{|l|}{ Humanities } \\
\hline $\begin{array}{l}\text { History } \\
\text { Other/mixed }\end{array}$ & $413.68(8)$ & $700.25(21)$ & $645.42(12)$ & $294.28(6)$ & $149.53(3)$ & $385.96(5)$ \\
\hline Total & $413.68(8)$ & $700.25(21)$ & $645.42(12)$ & $294.28(6)$ & $149.53(3)$ & $385.96(5)$ \\
\hline TOTAL & $\begin{array}{c}7037.59 \\
(210)\end{array}$ & $\begin{array}{c}5160.37 \\
(164)\end{array}$ & $\begin{array}{l}1972.28 \\
(67)\end{array}$ & $\begin{array}{c}3231.74 \\
(108)\end{array}$ & $\begin{array}{c}6198.48 \\
(166)\end{array}$ & $\begin{array}{c}6592.77 \\
(169)\end{array}$ \\
\hline
\end{tabular}

Table 2. Volume of science programs on VRT -TV1, 1997-2002 (in minutes and number of programs).

\begin{tabular}{|c|c|c|c|c|c|c|}
\hline Hard Sciences & 1997 & 1998 & 1999 & 2000 & 2001 & 2002 \\
\hline Aplied & $367.25(22)$ & $95.2(2)$ & & $160.56(4)$ & & $197.83(5)$ \\
\hline Natural & $3494.4(105)$ & $4438.4(101)$ & $4331.68(197)$ & $4069.53(413)$ & $2995.76(318)$ & $3003.46(121)$ \\
\hline Physical & $102.05(2)$ & $210.12(4)$ & & $413.9(9)$ & $157.36(4)$ & $703.58(15)$ \\
\hline Medical & $246.21(5)$ & & $768.55(16)$ & $1236.2(28)$ & $1705.73(51)$ & $2076.22(61)$ \\
\hline Other/mixed & $996.8(60)$ & $2248.3(51)$ & $3034.62(150)$ & $1771.58(134)$ & 2793.92(162) & $2630.7(181)$ \\
\hline Total & $5206.71(194)$ & 6992.02(158) & $8134.85(363)$ & 7651.77(588) & $7652.77(535)$ & $8611.79(383)$ \\
\hline \multicolumn{7}{|l|}{ Humanities } \\
\hline Literature \& Lang & g. $1759.68(112$ & 2) $598.05(39)$ & $550.56(39)$ & $36.05(1)$ & $91.68(2)$ & $190.86(3)$ \\
\hline History & $4077(99)$ & $1341.5(29)$ & $1855.13(40)$ & $3516.9(72)$ & $5800.7(160)$ & $5904.9(152)$ \\
\hline Other/mixed & $95.85(63)$ & & & $41.23(1)$ & $378.97(11)$ & $152.78(3)$ \\
\hline Total & $5932.53(274)$ & $1939.55(68)$ & $2405.69(79)$ & $3594.18(74)$ & $6271.35(173)$ & $6248.54(158)$ \\
\hline TOTAL & $\begin{array}{c}11139.24 \\
(468)\end{array}$ & $\begin{array}{c}8931.57 \\
(226)\end{array}$ & $\begin{array}{c}10540.54 \\
(442)\end{array}$ & $\begin{array}{c}11245.95 \\
(662)\end{array}$ & $\begin{array}{c}13924.12 \\
(708)\end{array}$ & $\begin{array}{c}14860.33 \\
(541)\end{array}$ \\
\hline
\end{tabular}

Table 3. The volume of science programs on VRT-TV2/Canvas/Ketnet, 1997-2002 (in minutes and number of programs). 
When we consider the volume of the different science domains, it becomes clear that TV1 focuses on hard sciences and in particular on natural sciences, which expand rapidly since 2000. Applied sciences and physical sciences have one production each on TV1 in 1997 but are since then completely ignored. The medical sciences that in 1997 were the second largest category are barely visible during the two following years and in 2000 they completely disappear. From the humanities we only find history, albeit even very inconstant and with only a few broadcasts. It may be stated that TV1 in this time period mainly broadcasts natural sciences and in addition a few historical programs.

Table 3 demonstrates that the second channel disposes of a wide range concerning both hard sciences and humanities. Yet the volume of applied and physical sciences on this channel is also minor. The medical sciences disappear for some time in 1998 but then keep increasing to 61 programs in 2002. Furthermore natural sciences are broadcast quite substantially on the second channel. During the period 1999-2000 we see a striking increase as opposed to a distinct decline in 2001-2002 when TV1 clearly selects natural sciences. Consequently both channels complement each other well. We also notice a large number of programs in the category 'Other or mixed hard science programs', which proves that quite a lot of programs on the second channel cannot be categorized easily. This was quite less the case on the first channel.

With respect to the main category of humanities on TV2/Canvas/Ketnet it can be stated that as opposed to the first channel the second station treats these better. The languages and literature subgroup decreases substantially and almost completely disappears from 2000 on. On the other hand we see a distinct increase of programs with a historical point of view. These primary differences urge upon a more profound analysis of the distinction between hard and human science programs.

\section{Volume of hard and human science programs on the VRT}

The volume of hard science programs (see table 4) completely serves the aforementioned general pattern: both an absolute and a relative decrease concerning the total broadcast hours from 1997 through 1999, followed in 2000 by an increase. The absolute figures show that there were 246.98 broadcast hours in 2002, substantially more than the 197.18 hours in 1997 . Yet in the view of the total broadcast hours of the public channel, the maximum share of hard science programs was in 1997 (3.40\%). Despite the increases in 2000, 2001 and 2002, the maximum share has never again been reached. The second channel shows a remarkable increase in hard science broadcasts between 1997 and 1999, with a slight decline in 2000 and 2001, and in 2002 again an increase of about 57 hours (from 86.78 hours to 143.53 hours).

\begin{tabular}{|llllcc|}
\hline & TV1(u) & TV2/Canvas/Ketnet(u) & VRT(u) & VRT (\%) & Total broadcast time (u) \\
$\mathbf{1 9 9 7}$ & 110.40 & 86.78 & 197.18 & 3.40 & 5797 \\
$\mathbf{1 9 9 8}$ & 74.33 & 116.53 & 190.86 & 2.86 & 6685 \\
$\mathbf{1 9 9 9}$ & 22.11 & 135.58 & 157.69 & 2.24 & 7026 \\
$\mathbf{2 0 0 0}$ & 48.96 & 127.53 & 176.49 & 2.47 & 7147 \\
$\mathbf{2 0 0 1}$ & 100.82 & 127.55 & 228.37 & 3.03 & 7549 \\
$\mathbf{2 0 0 2}$ & 103.45 & 143.53 & 246.98 & 2.99 & 8251 \\
\hline
\end{tabular}

Table 4. Length and share of hard science programs on vrt, 1997-2002 (in hours and percentages).

The volume of human science programs (see table 5) is quite small. Once again the same tendencies can be seen but we want to highlight two comments. Firstly, the humanities get less than half the airplay of their hard counterparts. A comparison of the absolute figures from 1997 (105.77 hours) and 2002 (110.57) shows the stagnation of the number of human science broadcast hours, as opposed to the hard science programs that show a relative increase of roughly $25 \%$ (197.18 hours in $1997,246,98$ hours in 2002). The second comment is that there is a really striking gap between the first and second channel: TV1 does not seem to program human sciences at all.

Next we will categorize the science programs according to target group, production method and finally in respect of the fact whether these programs are first-time productions or reruns, which allows an even more profound analysis of their profile and hence a better insight into the VRT's programming strategy. 


\begin{tabular}{|llclcc|}
\hline & TV1(h) & TV2/Canvas/Ketnet(h) & VRT(h) & VRT (\%) & Total broadcast time (h) \\
$\mathbf{1 9 9 7}$ & 6.89 & 98.88 & 105.77 & 1.82 & 5797 \\
$\mathbf{1 9 9 8}$ & 11.67 & 32.33 & 44 & 0.66 & 6685 \\
$\mathbf{1 9 9 9}$ & 10.76 & 40.09 & 50.85 & 0.72 & 7026 \\
$\mathbf{2 0 0 0}$ & 4.90 & 59.90 & 64.8 & 0.91 & 7147 \\
$\mathbf{2 0 0 1}$ & 2.49 & 104.52 & 107.01 & 1.42 & 7549 \\
$\mathbf{2 0 0 2}$ & 6.43 & 104.14 & 110.57 & 1.34 & 8251 \\
\hline
\end{tabular}

Table 5. Length and share of human science programs on vrt, 1997-2002 (in hours and percentages).

\section{Target groups}

Table 6 subdivides the volume of science programs according to target audience. We immediately notice the particular position of children and young people as a target group. At the start of the new programming strategy there is a sharp decline of the share of science programs targeted at the young, but then it increases from 16 hours in 1998 to 85.6 hours in 2001 . The majority of the science programs is intended for adults: in $200276 \%$ of all programs that can be categorized according to target groups are intended for adults, $19 \%$ for children and 5\% for families. The category of families has difficulties to find a niche for itself but since 2001 we see a certain minimum of science programs intended for families.

\begin{tabular}{|llcc|}
\hline & \multicolumn{2}{l}{ Kids (h) Adults (h) } & Families (h) \\
$\mathbf{1 9 9 7}$ & 49.6 & 252.9 & - \\
$\mathbf{1 9 9 8}$ & 16 & 217.4 & 0.4 \\
$\mathbf{1 9 9 9}$ & 33.8 & 173 & 2.1 \\
$\mathbf{2 0 0 0}$ & 48.1 & 193 & 0.9 \\
$\mathbf{2 0 0 1}$ & 85.6 & 227 & 22.4 \\
$\mathbf{2 0 0 2}$ & 67.7 & 270.7 & 17.8 \\
\hline
\end{tabular}

Table 6. Volume of science programs according to target groups, 1997-2002 (in hours).

\section{Production method}

With respect to production method (see table 7) the share of homegrown productions has been declining ever since the first year of our data. Currently it is impossible to say whether 2001 will be rock bottom and 2002 will be a turn. Both the share of co-productions and the volume of purchased productions have been gone up substantially, and particularly this last category makes up the majority of all science programs on the VRT since 1998. The new programming strategy apparently resulted in a cutback of own productions (from $69 \%$ down to $7.80 \%$ in 2001), in favor of the number of purchased programs (from $29.10 \%$ to $77.1 \%$ in 2001). The co-productions, which were barely broadcast in 1997, make up one quarter of all science programs in 2002. The question must be asked whether this is a cost-reducing (or split cost) strategy that turns up in the programming policy.

\begin{tabular}{|llcl|}
\hline & Own productions (\%) & Coproductions (\%) & Purchased (\%) \\
$\mathbf{1 9 9 7}$ & 69 & 1.90 & 29.10 \\
$\mathbf{1 9 9 8}$ & 40.60 & 2.30 & 57.10 \\
$\mathbf{1 9 9 9}$ & 22.40 & 18.10 & 59.50 \\
$\mathbf{2 0 0 0}$ & 8.30 & 14.80 & 76.90 \\
$\mathbf{2 0 0 1}$ & 7.80 & 15.10 & 77.10 \\
$\mathbf{2 0 0 2}$ & 10.60 & 23.40 & 66 \\
Total & $\mathbf{2 4 . 1 0}$ & $\mathbf{1 3 . 4 0}$ & $\mathbf{6 2 . 5 0}$ \\
& & & \\
\hline
\end{tabular}

Table 7. Production method (in percentages). 


\section{Type of broadcast}

Table 8 shows the figures with respect to the fact whether a program is a first-time production (hence being an 'original' program) or a rerun of a previous broadcast. We see the same shift as before: from 1997 through 1999 mainly original productions are broadcast while 2000 seems to be the starting point of a majority of reruns. Thus, from the moment that the public channel offers more science programs, the number of reruns has also gone up. This is the second factor to dim the possible euphoria with respect to the rebirth of sciences on the Flemish public channel. Table 9 subdivides these figures according to channel, in order to get a more profound analysis.

\begin{tabular}{|lcl|}
\hline & Original broadcast (\%) & Rerun (\%) \\
$\mathbf{1 9 9 7}$ & 67.3 & 32.7 \\
$\mathbf{1 9 9 8}$ & 83 & 17 \\
$\mathbf{1 9 9 9}$ & 75.2 & 24.8 \\
$\mathbf{2 0 0 0}$ & 28.1 & 71.9 \\
$\mathbf{2 0 0 1}$ & 51.5 & 48.5 \\
$\mathbf{2 0 0 2}$ & 37.4 & 62.6 \\
\hline
\end{tabular}

Table 8. Type of broadcast on VRT, 1997-2002 (in percentages).

Table 9 shows that the turn on TV1 only begins in 2001 and that the proportion of first-time broadcasts and reruns in 2002 is fifty/fifty again. Hence it is still premature to draw a permanent conclusion. However on the second channel the turn can already be noticed in the year 2000. The proportions in 2002 have not yet returned to their previous level, in spite of the near-normalization in 2001: two thirds of all science broadcasts on the second channel happen to be reruns. This means that on the main channel concerning science programming a lot more reruns are being broadcast since the year 2000 .

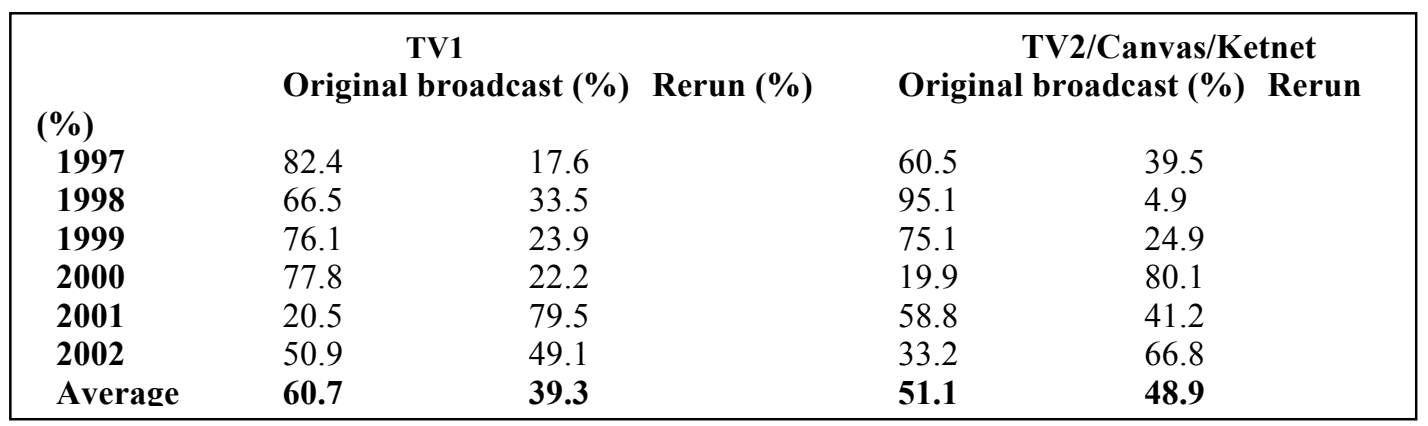

Table 9. Type of broadcast on VRT according to channel, 1997-2002 (in percentages).

\section{Some conclusions}

What is interesting about the previous analysis is that the public channel was reshaped in 1997 so that its annual results - in respect of the new programming strategy - clearly demonstrate the impact on science programming in Flanders. The annual figures show that the new mission and programming strategy result in both an absolute and relative decline of the proportion of science programs in the period 1997-1999. In the year 2000 an opposite movement starts and by 2002 we find about 55 hours more science programs than in 1997. These movements demonstrate a clear direction regarding channel policy and science domain. The gap between the broadening main channel and the investigative second channel gets deeper in the analyzed period. It can be concluded that the new programming strategy resulted in the main channel of the Flemish community focusing on natural sciences and the second channel - with respect to the humanities - focusing on history but also offering a wide range of hard science productions. These results confirm Göpfert ${ }^{3}$ who based on a comparative study of the UK and Germany predicted that in a highly competitive television landscape the main distinction regarding programming strategies will be 
found between the popular/broadening and specialized/investigative channels. Another outcome of the new programming strategy is the declining share of homegrown productions: these are replaced with mainly purchased programs and co-productions secondly.

Any feelings of euphoria concerning the rebirth in 2000 and the substantially higher offer of sciences in 2001 and 2002, however, must be dimmed based on two factors. Firstly, the public channel's total broadcast time has risen more than the broadcast hours of science programs, the result of which being that the relative share of sciences on the public channel has in fact decreased. Secondly, the figures clearly demonstrate that when the proportion of science programs starts to rise again, at the same time also the number of reruns is going up. Consequently it is premature to state that the VRT succeeded in stopping and reversing the downward spiral in 2000 with many new science productions.

\section{More science programs, various factors}

\section{Impact of the management contract}

The management contracts of both 1997-2001 and 2002-2006 stipulate that the VRT should "provide a high-quality selection of programs in the information, entertainment, education and culture sectors". A priority is the focus on "information and cultural programs aimed at the media user" and in addition to this there is also "sports, contemporary education, homegrown drama productions and entertainment". 12,13 Science programs are not specifically covered, they are categorized and measured based on the educational and exceptionally on the cultural performance standard which includes reaching on average $10 \%$ and $15 \%$ of the population, respectively. This performance standard is based on the number of Flemish persons aged four years or more that watches programs that are classified as educational or cultural at least 15 minutes per week. Figure 1 shows that the 10\% education norm was amply reached during our inquiry period and was particularly outstanding in 2001 and 2002. This means that in 2002 any educational program reached on average 1,606 million Flemish persons. The VRT annual report ${ }^{14}$ mentions the success of the program How? Like that! and the documentary series (such as OverLeven) on Canvas as an explanation for this. An initial decline is visible in 2005, notably because there are less How? Like that! episodes. The same paragraph of both management contracts explicitly states that programming should be aimed at specific population- and age groups, particularly children and young people. This demonstrates the specific position of that age group in addition to what is shown by the data (see 2001 and 2002).

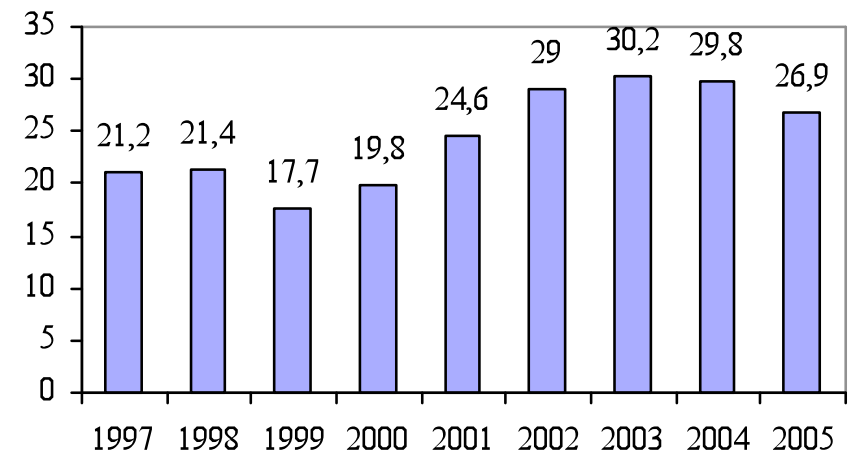

Figure 1. Cumulative weekly range of the educational VRT programs (in percentages).

The latest Management Contract 2007-2011 explicitly mentions Knowledge and Science as one of the new six production domains, with correspondent production units, of VRT, and stipulates that concerning educational programs $25 \%$ of the population should be reached. ${ }^{15}$ This evolution can be explained by both the tremendous success of science on television (especially How? Like that!) and a broader social discourse (see below). 


\section{Role of the Flemish government}

However, the management contract with the public channel is not the only argument in the Flemish authorities' pursuit to promote science programs on television: since 1994 the Flemish authorities have established a scientific and technological innovation policy with a particular emphasis on scientific information. The Flemish authorities have established a powerful instrument in order to "popularize science, technology and technological innovation"16 by means of annual action plans under the umbrella 'Science information and innovation'. The strategic objective behind it is "to enhance and maintain a social platform for science, technology and technological innovation". The annual action plans for their part should take care that the public 1) is informed about science, technology and research, 2) is made aware (of the impact) of this subject matter, 3) is given account of the government's science investments and 4) that a culture is created in which technological innovation can prosper. This means in particular to approach the overall audience and to stimulate its awareness. On the one hand, this has resulted in initiatives such as the Flemish Weeks of Science, the Science Fest or the Day of Technology. On the other hand, also the media are considered to be a vital means and hence since the year 2000 TV actions ('More Science on TV') are a part of the action plan based on the idea of the TV set - "albeit a superficial medium"17 - being the ultimate medium to reach the largest possible audience. The partnership agreement with the public channel resulted in programs such as OverLeven on Canvas and Curieuzeneuze on Ketnet on the one hand, and an agreement with the regional channels on the other. OverLeven was the only program still being produced in 2006 and because up to then there were no plans for new How? Like that! episodes, on July 17, 2006, the Department of Economy, Science and Innovation called on all Flemish channels to submit a program proposal regarding the popularization of science. ${ }^{18}$ The fact was that OverLeven was considered as failing to reach a large audience while the tremendous viewing figures of How? Like that! (see the substantial influence on the shift of the educational performance standard) had been noticed by the Flemish authorities. The final result of this appeal is that both the public channel and the commercial channels of the VMMA and SBS Belgium came with a positive reaction. This effectively meant the end of the blatant absence of science programs on Flemish commercial channels as in 2006 the VMMA broadcast Solar Team II with the support of the government.

\section{Social discourse}

In his historical analysis of science on the public channel Biltereyst ${ }^{1}$ describes how in the seventies and eighties a soft(er) science discourse arose in reaction to the absolute progress thinking of the sixties, which was characterized by a hard(er) science discourse. On television this translated into a shift from an emphasis on hard sciences to a growing interest in both history and social (and other human) sciences representing the more social and ethical dimensions of science and technology. During the last several years, from within a number of organizations, companies and the field of education, a renewed concern regarding the interest in sciences has been established, with a particular focus on how to encourage youngsters in this matter. Within the scope of the economic globalization of labor, companies are investigating the shifting of responsibilities in countries with high labor costs. Several reports have been published that indicate a structural lack of graduates in hard sciences. ${ }^{19,20,21}$ Bearing in mind the unexpected success of the midwifery and veterinary courses after docu-soaps in which these were the central activities and the great demand for entry kits for starting entrepreneurs after the TV soap about a business family, the Academy of Sciences and Technology Committee has urged to produce fiction series in the Flemish television landscape starring scientists, and if possible a researcher from a non-medical field who works on an invention or is about to force a breakthrough: "Preferably this would be an exciting story focusing on the dreams, setbacks, intrigues and successes of an inexhaustible scientist; and perhaps even sprinkled with a romantic touch". ${ }^{21}$ These elements indicate the rebirth of a (more) hard science discourse in Flemish society.

\section{Conclusion}

Clearly a new age has arrived for science television in Flanders. In 1999-2000 the VRT succeeded to reverse the downward spiral. In the first place this turn must be seen within the context of a traditional 
emphasis of public service philosophy on education - it is a substantial part of the public channel's social mission. It looks as if the VRT has made a virtue (the thorough reorganization with the new profiling of TV1 and the start of Canvas and Ketnet) of necessity (the arrival of a commercial competitor that took away a substantial market share) and established a solid internal policy regarding science television. A policy where even science productions are used to attract as many viewers as possible (see How? Like that! that was broadcast in prime time on Sunday evenings). The VRT did get generous help of the Flemish authorities who established a sound science policy containing a number of agreements with the public channel with respect to the popularization of science. This structural government support guaranteed the survival of science on TV and helped to boost science television in Flanders. And thirdly, the rise and strong representation of hard sciences on the public channel appears to reflect a social discourse that emphasizes scientific knowledge as the driving force of our socio-economic prosperity.

This evolution, however, needs to be annotated with a few comments. Our study showed that the turn was partly made possible because of the higher volume of purchased productions and reruns. Both elements are indicative for a cost-reducing strategy. Yet finally this proves that this does not necessarily obstruct an effective internal science policy.

With respect to future research it would be interesting to compare with other European broadcast channels by means of EBU codes on science programming, because these data constitute a uniform system of categories and allow long-term comparisons. This could also be supplemented with an inquiry into the categorization policy of other public channels that make use of alternative performance standards, which could influence the way that a program is categorized.

\section{Acknowledgements}

A previous version of this paper was prepared for the $10^{\text {th }}$ International Conference on the Public Communication of Science and Technology (PSCT-10) 'Bridges to the future' held at Malmö, Sweden, 23-27 June 2008. This study was supported by the Funds for Scientific Research - Flanders (FWOVlaanderen), G.0152.02N. We would like to thank the Dept. of Communication Studies at Ghent University in general, but also Ruben Dobbelaere and the VRT research group in specific for providing us with the data for this study. We are also grateful to Hans Verstraeten, Daniel Biltereyst, Lucien Hanssen, Ilse Devroe, Karolien Poels, and Stijn Joye for providing information or for fruitful comments on this paper. And lastly, we dedicate this paper to our beloved late colleague Frieda Saeys.

\section{Notes and references}

${ }^{1}$ D. Biltereyst, Wetenschappelijke televisieprogramma's: uitzending en waarneming. Televisie, publieke kennis en wetenschappen: de Vlaamse ervaring, Federale diensten voor wetenschappelijke, technische en culturele aangelegenheden, Brussel, 2001.

2 S. de Cheveigné and E. Veron (1996), Science on TV: Forms and reception of science programmes on French television, Public Understanding of Science 5(3): 231-253.

3 W. Göpfert (1669), Scheduled science: TV coverage of science, technology, medicine and social science and programming policies in Britain and Germany, Public Understanding of Science 5(4): 361-374.

${ }^{4}$ C.M. Koolstra, M.J.W. Bos and I.E. Vermeulen (2006), Through which medium should science information professionals communicate with the public: television or the internet?, Jcom 5(3): A01, Retrieved Oct 12, 2006; available at: $<\mathrm{http}: / /$ jcom.sissa.it/archive/05/03/Jcom0503(2006)A01/>

5 B. León (2008), Science related information in European television: a study of prime-time news, Public Understanding of Science 17(4): 443-460.

6 M. Long, G. Boiarsky and G. Thayer (2001), Gender and racial counter-stereotypes in science education television: A content analysis, Public Understanding of Science 10(3): 255-269.

7 P. Verhoeven (2008), Where has the doctor gone? The mediazation of medicine on Dutch television, 1961-2000, Public Understanding of Science 17(4): 461-472.

8 J. Willems and L. Hanssen (1992), Wetenschap op televisie: een vergelijking tussen vier Europese landen, Massacommunicatie 20(2): 128-138.

9 J. Willems and L. Hanssen (1993), Science and Technology on TV-Four European Countries compared, Communications 18(2): 215-221

${ }^{10}$ Interview Mark Van Lombeek, May 11, 2004.

${ }^{11}$ Vlaams Parlement, Decreet betreffende de omzetting van de BRTN in een naamloze vennootschap van publiek recht, April 29 , 1997. 


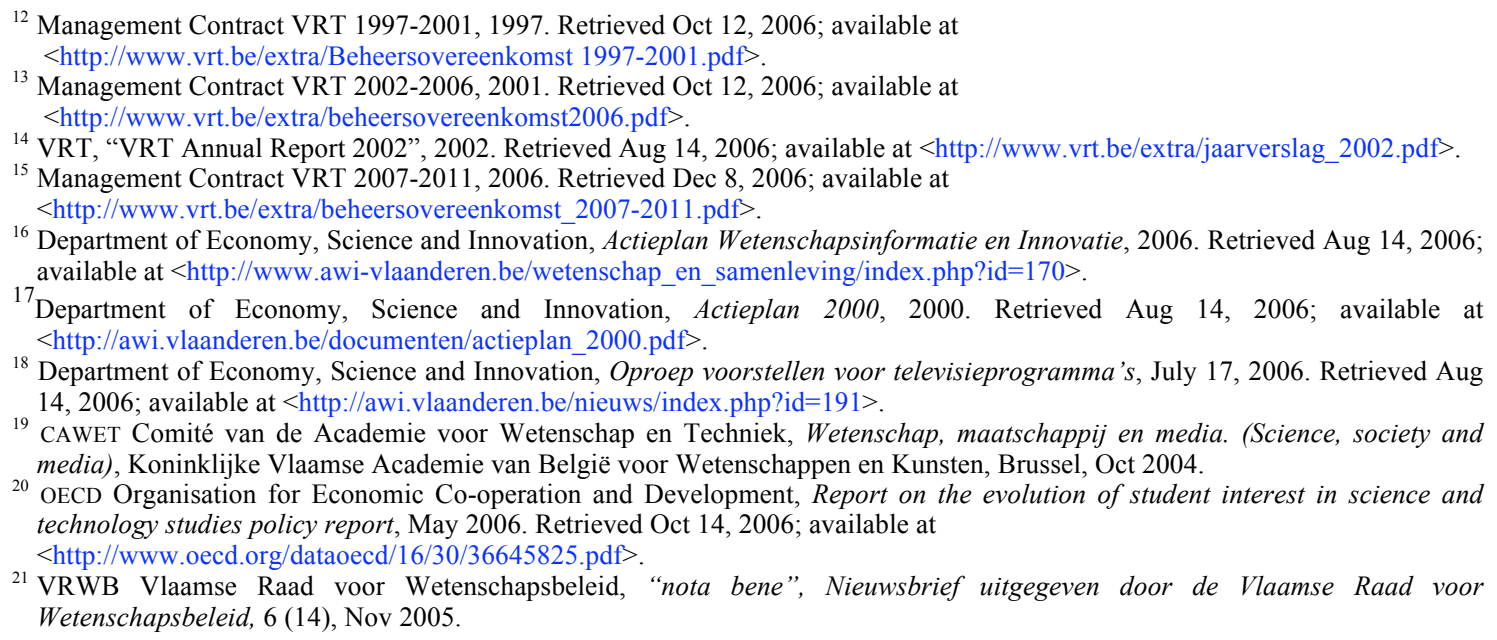

\section{Author}

Dr. Pieter Maeseele is a lecturer of Media Sociology, International Communication and Intercultural Communication at Erasmus University College Brussels. He is also affiliated as a researcher to the Centre for Cinema and Media Studies (CIMS) and the Centre for Critical Philosophy (CCP) at Ghent University. As a media sociologist, his research mainly focuses on the representation and reception of science, technology and the environment in the media. Correspondence to Pieter Maeseele, Kortrijkse Heerweg 110, 8540 Deerlijk, Belgium. E-mail: Pieter.Maeseele@Gmail.com.

Dr. Lieve Desmet is a lecturer at Erasmus University College Brussels and her research focuses on the history of news. E-mail: Lieve.Desmet@ehb.be.

How TO CITE: $\quad$ P. Maeseele and L. Desmet, Science on television: how? Like that!, Jcom 08(04) (2009) A03. 